\title{
Relação entre Tumores Ovarianos Epiteliais Borderline e Francamente Invasores: Epidemiologia, Histologia e Prognóstico
}

\author{
The Relationship between Borderline and Invasive Epithelial Ovarian Tumors: \\ Epidemiology, Histology and Prognosis
}

Sophie F. Mauricette Derchain ${ }^{1}$, José Carlos Campos Torres ${ }^{1}$, Luiz Carlos Teixeira ${ }^{1}$, Liliana A. Lucci de Angelo Andrade ${ }^{2}$, Frederico Ken Miyahara Masuko ${ }^{1}$, Marcos Antônio Santos ${ }^{1}$

\section{RESUMO}

Objetivo: avaliar alguns aspectos epidemiológicos, do diagnóstico e do prognóstico em mulheres com tumores epiteliais ovarianos borderline e francamente invasores.

Métodos: foram revisados os prontuários de 198 pacientes tratadas no CAISM/UNICAMP de 1986 a 1996. Para análise estatística foram utilizados os testes $\chi^{2}$, exato de Fisher, $t$ de Student e curvas de sobrevida pelo método de Kaplan-Meyer comparadas pelo teste logrank. O seguimento médio das pacientes foi de 50 meses (de 11 a 168). Dos 198 casos, 24 eram tumores borderline (12\%) e 174 (88\%) carcinomas francamente invasores.

Resultados: a média de idade das pacientes com tumores borderline foi significativamente menor que a das mulheres com carcinoma francamente invasor: $43 \pm 14,8$ anos vs $52 \pm 12,6$ anos ( $p<0,002)$. Os tipos histológicos mais freqüentes foram serosos (81 casos - 41\%) $e$ mucinosos (46 casos - 23\%). As mulheres com tumores borderline tiveram sua doença diagnosticada em estádios mais precoces $(p<0,0001)$. A biópsia de congelação, realizada em 77 pacientes, mostrou uma boa concordância com a biópsia de parafina nos casos de carcinoma francamente invasor. Entretanto, nos tumores borderline a taxa de erro foi alta (13\%), sendo que a maioria das falhas diagnósticas da congelação ocorreu entre os tumores mucosos. Em relação ao prognóstico, a taxa de sobrevida foi significativamente maior nas pacientes com tumores borderline $(p<0,001)$.

Conclusões: as pacientes com tumores epiteliais ovarianos borderline foram mais jovens que aquelas com tumores francamente invasores, apresentaram a doença em estádios iniciais e tiveram melhor prognóstico quando comparadas àquelas com carcinoma francamente invasor.

PALAVRAS-CHAVE: Ovário: câncer. Epidemiologia. Tumores de malignidade limitrofe.

\section{Introdução}

Os tumores borderline, responsáveis por $5 \%$ a $20 \%$ dos tumores epiteliais malignos do ovário ${ }^{1}$,

${ }^{1}$ Área de Oncologia do Departamento de Tocoginecologia

${ }^{2}$ Departamento de Anatomia Patológica

Faculdade de Ciências Médicas da Universidade Estadual de Campinas (UNICAMP)

Correspondência:

Sophie F.M. Derchain

Rua Dr António Hossri, 629

Cidade Universitária

13083-140 - Campinas - SP

Fone/Fax: (019) 287-5538

Apoio financeiro para o trabalho FAPESP: número 97 04545/7 foram inicialmente descritos por Taylor em 1929. Foram reconhecidos como uma entidade patológica pela Federação Internacional de Obstetrícia e Ginecologia (FIGO) ${ }^{2}$ e pela Organização Mundial da Saúde na década de $70^{13}$. Podem ser denominados "cistoadenoma papilar hiperplásico" ${ }^{14}$ ou de "baixo potencial maligno" $5,6,7$ e aparentemente originamse dos ductos mullerianos ${ }^{8}$. Quando comparados com os carcinomas francamente invasores, os tumores borderline apresentam um comportamento menos agressivo, são diagnosticados em estádios mais precoces e acometem mulheres mais jovens $^{3,5,6,7}$. 
Histologicamente, o tumor é caracterizado por uma proliferação epitelial na ausência de invasão do estroma. Apresenta proliferação celular maior do que o encontrado no tipo benigno do mesmo tumor, representada por papilas complexas com células que tendem a se destacar para a luz dos cistos; epitélio estratificado em várias camadas celulares, aumento da atividade mitótica e, finalmente, atipia celular leve ou moderada $^{2}$. Os tipos histológicos mais comuns e melhor estudados são os serosos e os mucinosos ${ }^{14}$. Dentro de um mesmo tumor pode-se encontrar áreas de aspectos benignos, borderline e malignos, principalmente no tipo histológico mucinoso. Recentemente, tem-se dado particular ênfase ao estudo dos implantes peritoneais, invasivos ou não, que acompanham os tumores borderline, aos focos de microinvasão estromal e à presença de metástases linfonodais ${ }^{5,9}$. De uma forma geral, os tumores borderline são considerados carcinomas, sendo assim estadiados conforme as orientações da FIGO. Entretanto, têm um prognóstico muito melhor que os carcinomas francamente invasores ${ }^{1,4,14,15}$. Assim, os procedimentos cirúrgicos necessários para estadiamento e tratamento complementar são controversos ${ }^{3,5,6,8,9}$.

O objetivo deste estudo foi avaliar alguns aspectos epidemiológicos, do diagnóstico e do prognóstico em mulheres com tumores borderline de ovário, comparando-as com pacientes com carcinoma francamente invasor.

\section{Material e Métodos}

Este é um estudo clínico retrospectivocomparativo realizado com a revisão dos prontuários de 198 pacientes com carcinoma de ovário atendidas e tratadas no Centro de Atenção Integral à Saúde da Mulher (CAISM) da Universidade Estadual de Campinas (UNICAMP) de janeiro de 1986 a janeiro de 1996, com seguimento até dezembro de 1997. O seguimento médio das pacientes foi de 50 meses (de 11 a 168). Foram avaliados: idade, paridade, tipo histológico, diagnóstico obtido na biópsia intraoperatória de congelação, estádio e sobrevida de todas as pacientes. Todas as mulheres foram submetidas à cirurgia para estadiamento e citorredução, quando possivel. Em relação à avaliação histológica, todos os casos foram avaliados pelo Departamento de Anatomia Patológica da Faculdade de Ciências Médicas da UNICAMP e para diagnóstico de tumor borderline, ou francamente invasor, foram utilizados os critérios de Serov e colaboradores ${ }^{12}$. Das 174 mulheres com carcinoma francamente invasor, 149 foram submetidas à quimioterapia com cisplatina ou carboplatina associada à ciclofosfamida (seis ciclos em média). As demais 23 mulheres com carcinoma francamente invasivo apresentavam a doença em estádio Ia e não foram submetidas ao tratamento antiblástico. Das 24 mulheres com tumor borderline do ovário, sete foram submetidas à quimioterapia por meio do mesmo esquema, sendo duas por doença estádio Ic e cinco por doença estádio II/III. Para análise estatística foram utilizados os testes $\chi^{2}$, exato de Fisher e t de Student, e foram realizadas curvas de sobrevida pelo método de Kaplan-Meyer comparadas pelo teste log-rank, com limite de confiança de 95\%.

\section{Resultados}

Das 198 pacientes avaliadas, 24 (12\%) correspondiam a tumores borderline e 174 (88\%) eram carcinomas francamente invasores. A idade média das pacientes com tumores borderline foi de $43 \pm 14,8$ anos e das pacientes com carcinomas francamente invasores foi de $52 \pm 12,6$ anos, sendo esta diferença estatisticamente significativa $(p<0,002)$. Quanto à paridade não houve diferença entre os grupos. Houve maior incidência dos tipos serosos e mucinosos: 12 pacientes com tumores borderline e 69 daquelas com carcinomas francamente invasores apresentavam tumores serosos e 11 tumores borderline e 35 carcinomas francamente invasores eram mucinosos. Uma paciente com tumor borderline e 33 com carcinoma francamente invasor apresentavam tumores endometrióides e, finalmente, encontraram-se entre os carcinomas francamente invasores, $10 \mathrm{de}$ células claras e 27 carcinomas indiferenciados. A doença diagnosticada em estádios iniciais foi significativamente mais freqüente nas pacientes com tumores borderline $(\mathrm{p}<0,0001)$ e a presença de células malignas na citologia do lavado peritoneal foi significativamente mais freqüente naquelas com carcinomas francamente invasores $(\mathrm{p}=0,05)($ Tabela 1$)$.

O estudo histológico no intra-operatório com a técnica de congelação foi realizada em 77 pacientes. A concordância entre os diagnósticos obtidos no intraoperatório e os com parafina ocorreu em 67 casos: 11 tumores borderline e 56 carcinomas francamente invasores. Dentre as 63 pacientes com diagnóstico definitivo de carcinoma francamente invasor, $4(6 \%)$ haviam sido classificados como borderline na congelação e $3(4,5 \%)$ como benigno, dos quais um como endometriose. Dos 14 tumores considerados borderline na análise do material parafinado, dois haviam sido classificados como benignos no exame por congelação e um como francamente invasor. Assim, a proporção de classificação incorreta no intraoperatório foi de 13\% (Tabela 2). 
Tabela 1 - Distribuição das pacientes com tumores ovarianos "borderline" e francamente invasores segundo a idade, paridade, tipo histológico e extensão da doença.

\begin{tabular}{lccccc}
\hline Variáveis & \multicolumn{4}{c}{ Tumores borderline } & \multicolumn{2}{c}{ Invasores } & $\mathbf{p}$ \\
\hline Idade & & & & & \\
$<30$ anos & 4 & $(16 \%)$ & 9 & $(5 \%)$ & \\
$30-45$ anos & 10 & $(42 \%)$ & 36 & $(21 \%)$ & \\
$>45$ anos & 10 & $(42 \%)$ & 129 & $(74 \%)$ & \\
Idade média & $43 \pm 14,8$ anos & $52 \pm 12,6$ anos & 0,002
\end{tabular}

Paridade

$\begin{array}{lrrrrr}\text { Nulípara } & 7 & (29 \%) & 36 & (21 \%) & \\ \text { 1 a 2 partos } & 6 & (25 \%) & 83 & (48 \%) & \\ \text { 3 partos ou mais } & 11 & (46 \%) & 55 & (31 \%) & 0,1\end{array}$

Tipo histológico

$\begin{array}{lrllll}\text { Seroso } & 12 & (50 \%) & 69 & (40 \%) & \\ \text { Mucoso } & 11 & (46 \%) & 35 & (20 \%) & \\ \text { Endometrióide } & 1 & (4 \%) & 33 & (19 \%) & \\ \text { Células claras } & 0 & & 27 & (15 \%) & \\ \text { Indiferenciados } & 0 & & 10 & (6 \%) & \text { Não se aplica }\end{array}$

\section{Estádio}

$\begin{array}{lrlrll}\text { I } & 19 & (79 \%) & 59 & (34 \%) & \\ \text { Ia } & 16 & & 44 & & \\ \text { Ib } & - & & 2 & & \\ \text { IC } & 3 & & 13 & & \\ \text { II } & 3 & (12,5 \%) & 7 & (4 \%) & \\ \text { III } & 2 & (8,5 \%) & 89 & (51 \%) & \\ \text { IV } & 0 & & 19 & (11 \%) & <0,0001\end{array}$

Citologia peritoneal

$\begin{array}{lrlcll}\text { Negativa } & 15 & (63 \%) & 71 & (41 \%) & \\ \text { Positiva } & 1 & (4 \%) & 31 & (18 \%) & \\ \text { Ignorado } & 8 & (33 \%) & 72 & (41 \%) & 0,05\end{array}$

TOTAL 24

174

Tabela 2 - Comparação entre os diagnósticos obtidos com a biópsia de congelação intraoperatória e a obtida com análise do material parafinado nas pacientes com tumores borderline e invasivos de ovário.

\section{Biópsia de congelação}

\begin{tabular}{lccc} 
Diagnóstico definitivo & Borderline & Invasor & Benigno \\
\hline Borderline & 11 & 1 & 2 \\
Invasor & 4 & 56 & $3^{*}$ \\
Benigno & $1^{* *}$ & 0 & 0 \\
TOTAL (N) & & & \\
\hline
\end{tabular}

* O diagnóstico em um dos casos foi endometriose.

** Excluído da casuística.
Em relação ao prognóstico, 22 mulheres (92\%) com tumores borderline e 85 (49\%) daquelas com carcinoma invasor estavam sem doença na última consulta (Tabela 3) e a taxa de sobrevida foi significativamente maior $(\mathrm{p}<0,001)$ para as pacientes com tumores borderline (Figura 1).

Tabela 3 - Distribuição das pacientes com tumores borderline e francamente invasores do ovário segundo a evolução.

\begin{tabular}{lrrrr}
\hline Evolução & \multicolumn{2}{c}{ Tumores borderline } & \multicolumn{2}{c}{ Carcinomas invasores } \\
& \multicolumn{1}{c}{ N $^{\circ}$} & \multicolumn{1}{c}{$\%$} & \multicolumn{1}{c}{ N $^{\circ}$} & \multicolumn{1}{l}{$\%$} \\
\hline Viva e sem doença & 22 & $92 \%$ & 85 & $49 \%$ \\
Viva e com doença & 2 & $8 \%$ & 36 & $21 \%$ \\
Óbito & 0 & & 53 & $30 \%$ \\
TOTAL (N) & 24 & & 174 & \\
\hline
\end{tabular}

$(p<0,001)$

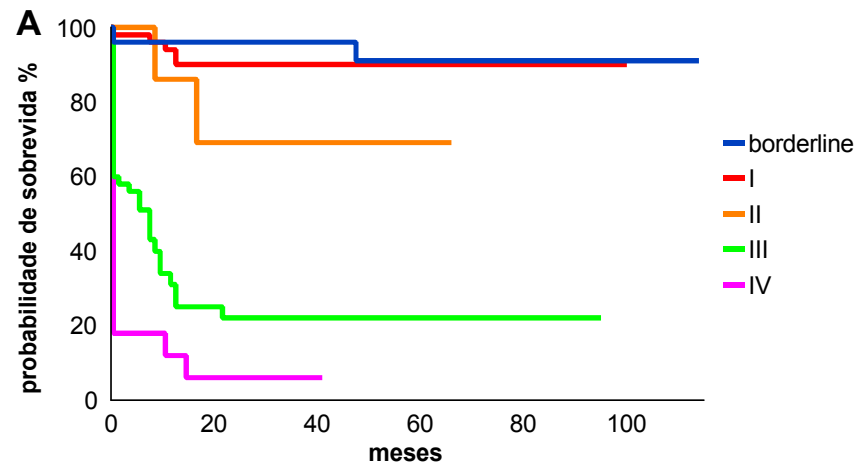

(A) Sobrevida livre de doença

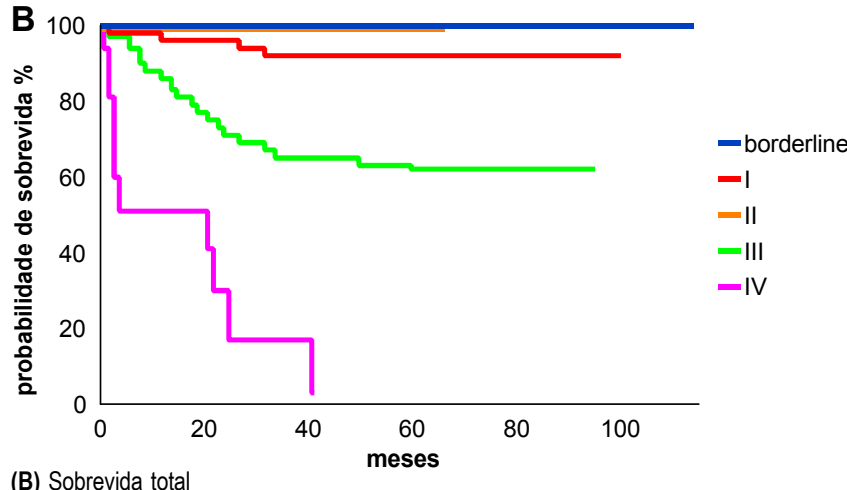

Figura 1 - Taxa acumulada de sobrevida livre de doença (A) e sobrevida total (B) das pacientes com tumores borderline e invasivos do ovário por estádios.

\section{Discussão}

Entre 1986 e 1996, 12\% dos tumores epiteliais malignos do ovário atendidos no serviço de Oncologia Genital do Departamento de 
Tocoginecologia da UNICAMP foram classificados como borderline. A média de idade destas pacientes na época do diagnóstico foi de 43 anos, dez menos que as pacientes descritas por Ji et al. ${ }^{3}$, na Finlândia, e semelhante ao referido no estudo de Tamakoshi et al. ${ }^{13}$, no Japão. Quase $60 \%$ das pacientes com tumores borderline apresentavam idade inferior a 45 anos, porém, apenas $16 \%$ tinham menos de 30 anos, justificando-se, assim, a paridade semelhante nos dois grupos.

Os tumores dos tipos histológicos seroso e mucinoso foram mais freqüentes entre os tumores borderline e houve apenas um caso com tumor borderline endometrióide. Os tumores endometrióides borderline são raros e seu comportamento biológico não é totalmente conhecido. A avaliação histológica de invasão estromal do tumor primário ou dos implantes pode ser dificil na presença de estruturas glandulares e irregulares em um estroma imaturo e edemaciado. Os tumores borderline serosos são mais freqüentemente bilaterais que os mucosos, os quais porém tendem a ser maiores ${ }^{3,8,13}$. Nesta série os três tumores borderline no estádio Ic e quatro dos cinco no estádios II/III eram serosos.

A biópsia intra-operatória pela técnica de congelação apresentou uma dificuldade para qualificar os tumores borderline. A biópsia de congelação intra-operatória apresenta classicamente uma baixa sensibilidade, principalmente nos tumores mucosos onde os resultados inadequados chegam a $30 \%{ }^{11}$. Como os tumores borderline ocorrem em mulheres jovens, nas quais a probabilidade dos tumores de ovário serem benignos também é maior, a avaliação do tumor ou do anexo tumoral em parafina é ideal. Se a neoplasia for borderline ou francamente invasora é melhor indicar uma nova laparotomia para estadiamento ou tratamento. A extensão da cirurgia completa para estadiamento - biópsias múltiplas, omentectomia, apendicectomia e linfadenectomia retroperitoneal nos tumores borderline de ovário é controversa. A indicação da apendicectomia e omentectomia se justifica nos tumores mucinosos do ovário pela possivel associação com o pseudomixoma peritoneal ${ }^{10}$. Já a invasão linfonodal é rara e sua relação com a sobrevida não está bem definida. Nesta casuística a linfadenectomia retroperitoneal foi realizada em 11 casos e todos os linfonodos estavam livres de neoplasia. A presença de doença linfonodal é descrita em até $21 \%$ dos casos referidos na literatura, porém pode ser considerada uma transformação mesotelial in situ no linfonodo quando não se encontra nenhum foco de invasão no ovário ${ }^{8}$.

No período estudado, $79 \%$ das pacientes com tumores borderline foram classificadas como estádio I, dados comparáveis com outros autores ${ }^{3,8,13}$. Entretanto, a cirurgia conservadora com preservação do útero e do ovário contralateral somente foi realizada em quatro casos. Ji et al. ${ }^{3}$ referem uma melhora da qualidade de vida em pacientes jovens submetidas à cirurgia conservadora, apesar da maior taxa de recidiva tumoral, principalmente no ovário contralateral, quando comparada com as pacientes submetidas à cirurgia radical. A maioria dos autores sugere que a cirurgia conservadora seja realizada em mulheres jovens que desejem completar a prole e apresentem a doença restrita a um ovário ${ }^{3,13}$. Pacientes mais idosas ou com prole completa podem ser submetidas à cirurgia radical para estadiamento adequado.

A quimioterapia antiblástica foi utilizada em sete pacientes com tumores borderline estádios Ic, II e III. Foram documentadas respostas clínicas em pacientes com tumores borderline avançados, quando submetidas aos esquemas contendo platina. A platina e seus derivados, são, sem dúvida, a terapia antiblástica de maior importância no tratamento dos tumores ovarianos, embora seu papel nos tumores borderline ainda não esteja bem definido ${ }^{8,13}$. Independentemente da cirurgia e quimioterapia, a sobrevida das pacientes com tumores borderline - mesmo agrupando-se os estádios I, II e III - foi significativamente melhor que aquela observada em pacientes com tumores francamente invasores ${ }^{14}$.

Concluiu-se que as pacientes com tumor borderline de ovário eram mais jovens, a maioria apresentava doença em estádios iniciais e tiveram um melhor prognóstico quando comparadas aos carcinomas invasivos. Apesar da biópsia de congelação de ovário ser um exame de boa acurácia em mulheres com tumores epiteliais francamente invasores, essa acurácia diminui nos tumores "borderline". Nesses casos, sugere-se aguardar o diagnóstico anatomopatológico definitivo em parafina. Se for confirmado tumor borderline, avaliar a extensão da doença e a idade da paciente para indicar a cirurgia radical.

\section{SUMMARY}

Purpose: to determine some epidemiological, diagnostic and prognostic aspects in women with borderline and invasive epithelial ovarian tumors.

Methods: the charts of 198 women treated at CAISM/ UNICAMP from 1986 to 1996 were revised. For statistical 
assessment, $\chi^{2}$, Fisher's exact and $t$ Student's tests were used when appropriate, followed by survival curves by the KaplanMeyer method, compared by the log-rank test. The mean follow-up was 50 months (11 to 168).

Results: the overall rate of borderline tumors was 12\% (24 cases), and for invasive carcinoma, 88\% (174 cases). The mean age of the patients with borderline tumors was significantly lower than that of those with invasive carcinoma $(43 \pm 14.8$ years vs. $52 \pm 12.6$ years, $p<0.002)$. The most frequent histologic types were the serous (81 cases: $41 \%$ ) and the mucinous (46 cases: 23\%) tumor. The women with borderline tumors had their diseases diagnosed in earlier stages when compared with the invasive carcinoma patients $(p<0.0001)$. The frozen biopsy, performed on 77 patients, showed a high agreement with the paraffin fixed tissue in the invasive carcinoma cases. However, in borderline tumors, the rate of failure was higher (13\%) and the major rate of failure was in mucinous tumors. Regarding prognosis, the survival rate was significantly higher in borderline tumors $(p<0.001)$.

Conclusions: women with epithelial ovarian tumors were younger, presented the disease at earlier stages, and had a better prognosis when compared with those with invasive carcinoma.

KEY WORDS: Ovarian neoplasia. Epidemiology. Borderline tumors.

\section{Referências Bibliográficas}

1. Gotlieb WH, Flikker S, Davidson B, Korach Y, Kopolovic J, Ben-Baruch G. Borderline tumor of the ovary. Fertility treatment, conservative management, and pregnancy outcome. Cancer 1998;82:141-6.

2. International Federation of Gynecology and Obstetrics. Annual report on the results of treatment in gynecologic cancer. Int J Gynaecol Obstet 1993, 28:189-90.

3. Ji H, Yliskoski M, Anttila M; Syrjänen K, Saarikoski S. Management of stage I borderline ovarian tumors. Int J Gynaecol Obstet 1996; 54:37-44.
4. Kehoe S, Powell J. Long-term follow-up of women with borderline ovarian tumors. Int $\mathrm{J}$ Gynecol Obstet 1996; 53:139-43.

5. Kennedy AW, Hart WR. Ovarian papillary serous tumors of low malignant potencial (serous borderline tumors). A long-term follow-up study, including patients with microinvasion, lymph node metastasis, and transformation to invasive serous carcinoma. Cancer 1996; 78:278-86.

6. Kurman RJ, Trimble CL. The behavior of serous tumors of low malignant potencial: are they ever malignant? Int J Gynecol Pathol 1993; 12:120-7.

7. Link CJ Jr, Kohn E, Reed E. The relationship between borderline ovarian tumors and epithelial ovarian carcinoma: epidemiologic, pathologic, and molecular aspects. Gynecol Oncol 1996; 60:34754.

8. Link CJ Jr, Reed E, Sarosy G, Kohn EC. Borderline ovarian tumors. Am J Med 1996; 101:217-25.

9. Michael H, Roth LM. Invasive and noninvasive implants in ovarian serous tumors of low malignant potencial. Cancer 1986; 57: 1240-7.

10.Ronnet BM, Kurman RJ, Zahn CM, Shmookler BM, Jablonski KA, Kass ME, et al. Pseudomixoma peritonei in women: a clinicopathologic analysis of 30 cases with emphasis on site of origin, prognosis, and relationship to ovarian mucinous tumors of low malignant potencial. Hum Pathol 1995; 26: 509-24.

11.Rose PG, Rubin RB, Nelson BE, Hunter RE, Reale FR. Accuracy of frozen-section (intraoperative consultation) diagnosis of ovarian tumors. Am J Obstet_Gynecol 1994;171:823-6.

12.Serov SF, Scully RE, Sobin LH. Histological typing of ovarian tumours. International histological classification of tumours, $\mathrm{n}^{\circ}$ 9, Geneva; World Health Organization, 1973.

13.Tamakoshi K, Kikkawa F, Nakashima N, Tamakoshi A, Kawai M, Furuhashi Y, et al. Clinical behavior of borderline ovarian tumors: a study of 150 cases. J Surg Oncol 1997; 64: 147-52.

14.Tropé C \& Kaern J. Prognosis and management of borderline tumours of the ovary. Curr Opin Obstet Gynecol 1996; 8:12-6. 